\title{
Novo protocolo para as ações de saúde bucal coletiva: padronização no armazenamento, distribuição euso do material de higiene bucal
}

\section{A new protocol for use and storage of tooth-brushing material for school children}

Fabiano Vieira Vilhena ${ }^{1}$

Silvia H elena de Carvalho Sales-Peres ${ }^{1}$

Magali deLourdes Caldana ${ }^{1}$

M arília Afonso Rabelo Buzalaf ${ }^{1}$

${ }^{1}$ Departamento de Odontopediatria, OrtodontiaeSaúde Coletiva, Faculdade de Odontologia deBauru, Universidade de São Paulo. Al. Dr. Octávio Pinheiro Brisolla 975, Vila Universitária. 17043-101 Bauru SP.

escovinha@escovinha.com.br
Abstract The aim of this study was to evaluate the storage and distribution of toothbrushing material for school children. Twenty individuals responsiblefor the collective oral hygiene of school children from Bauru - SP and São José dos Campos-SP participated in the first stage of thestudy, answering 2 questionnaires about five different kits for use by school children. Thestatistical analysis was performed using Wilcoxon $(p<0,05)$. In the second stage, the amount of toothpaste or liquid cleanser applied to the toothbrush by 178 school children aged 4 to 8 years from 2 cities from the state of São Paulo (Bauru e Bariri) was weighed using a portable balance. The statistical analysis was obtained by using Pearson's correlation coefficient and analysis of covariance ( $p$ $<0,05)$. Kit 5 obtained levels of satisfaction and high satisfaction when compared with the others kits (1 - 4). The school children from Bauru $(0,41 \mathrm{~g})$ used smaller amounts of toothpaste than the school children from Bariri $(0,48 \mathrm{~g})$. The average of the amount of liquid cleanser applied by the sample was $0,15 \mathrm{~g}$. The "drop technique" (liquid cleanser) was considered practical for dispensinga small, standardized quantity of the product. $K$ it 5 was considered a good alternative for establishing a collective oral health protocol in the Brazilian health system.

Key words Toothpaste, Fluorosis, Preventive dentistry, M aterials
Resumo Este trabalho avaliou o material de higiene bucal usado em escolas para estabelecer um protocolo às ações de higiene bucal coletiva. 0 estudo foi dividido em duas etapas: $1^{\text {a }} 20$ responsáveis pelos procedimentos coletivos com escolares de Bauru e São José dos Campos - SP responderam a dois questionários sobre o uso de cinco kits de higiene bucal coletiva. A análise estatística foi realizada através do testeWilcoxon $(p<0,05)$; $2^{\text {a }}$ - 178 escolares de 4 a 8 anos de Bauru e Bariri - SP dispensaram na escova uma quantidade de creme dental e dentifrício líquido para a prática da escovação, a qual foi pesada através de uma balança portátil. A análise estatística foi obtida através do coeficiente de correlação de Pearson ea análise de covariância $(p<0,05)$. 0 kit 5 obteve graus desati sfação e muita satisfação quando comparado aos kits 1 a 4 . A quantidade de creme dental dispensada pelos escolares foi em média $0,41 \mathrm{~g}$ (Bauru) e 0,48g (Bariri). Não houve diferença estatística entre os escolares de Bauru e Bariri em relação ao dentifrício líquido (média de 0,15g). 0 dentifrício líquido, através da "técnica da gota", foi considerado prático, dispensando uma peque na quantidade padronizada. 0 kit 5 demonstrou ser uma boa alternativa ao estabelecimento deum protocolo de ações em saúde bucal coletiva no SU S. Palavras-chave Dentifrício, Fluorose, Odontologia preventiva, M ateriais 
Introdução

É de conhecimento de gestores, administradorese técnicos do serviço público odontológico que as ações de saúde bucal coletiva são de extrema importância para o sucesso de programas de saúde dentro do Sistema Ú nico de Saúde (SUS). Dessa maneira, a higiene bucal coletiva diária nos espaços sociais deve ser amplamente preconizada ${ }^{1-5}$. Contudo, esta prática não deveria se resumir ape nas na distribuição de escova e dentifrícios aos escolares. A escova eo creme dental são materiais de uso individual e acabam sendo acondicionados e utilizados de forma coletiva sem as devidas condições higiênicas esanitárias ${ }^{6}$. A falta deconhecimento dos responsáveis, e principalmente a fal ta de material adequado para o armazenamento, são os principais motivos dos "insucessos" nas ações coletivas de prevenção odontológica6-8. Em 1989, Dusablon et al. identificaram as principais características de um kit de higiene bucal usado em ambientes coletivos que pudessem prevenir doenças contagiosas transmitidas entre as crianças que passam o dia todo em creches. Em conclusão, um dispositivo para o armazenamento deste material deve ter quatro requisitos: (1) fácil de ser construído; (2) baixo custo; (3) permitir o armazenamento de 15 escovas; (4) permitir a fácil identificação da escova pela criança e pelo professor ${ }^{9}$.

Um protocolo de ações deve ser estabelecido em relação à administração e acondicionamento dos materiais de higiene bucal coletiva, para que assim uma padronização possa ser adotada no SUS ${ }^{6}$.

Além do acondicionamento, outro fator importante é o uso racional do flúor devido ao aumento da prevalência defluorose dentária. A fluorose é um distúrbio de caráter dose-dependente, apresentando um aspecto clínico diretamente relacionado à quantidade de $\mathrm{F}$ absorvido pelo indivíduo. Uma vez que a fluorose ocorre durantea amel ogênese, a idade derisco para a dentição permanente éde 11 meses a sete anos de idade ${ }^{10}$.

Um dos fatores atribuídos ao desenvolvimento da fluorose éo uso precoce de dentifrícios fluoretadose, conseqüentemente, a sua ingestão pela população infantil ${ }^{10-15}$.

Tendo em vista estas considerações, fica evidente que medidas para reduzir a ingestão de $\mathrm{F}$ pelas crianças são necessárias, pois desde que 0 dentifrício passou a ter flúor em sua composição, o mesmo deixou de ter apenas a função cosmética eassumiu a função terapêutica ${ }^{5,15}$. 0 dentifrício como agente terapêutico deve ser dosado como todos os medicamentos.
A proposta deste trabalho foi avaliar formas de acondicionamento e distribuição do material dehigienebucal (kitscoletivos) utilizado em escolas e creches, relacionando as questões de armazenamento, higiene, praticidadeecusto. Além disso, procurou-se encontrar um material que possa ser padronizado e que atenda as normas de vigilância sanitária, para que dessa maneira um protocolo deações em higienebucal coletiva possa ser elaborado.

\section{Métodos}

O protocolo deste estudo foi aprovado pelo Comitê de Ética e Pesquisa da Faculdade de O dontologia de Bauru (protocolo $n^{\circ}$ 92/2004), sendo o consentimento livre esclarecido assinado pelos pais e ou responsáveis de todos os participantes.

Foram avaliados cinco kits de higiene bucal coletiva: kit 1 - estojo individual de plástico com tampa $(20 \mathrm{~cm} / 7 \mathrm{~cm} / 3 \mathrm{~cm})$, identificação do material feita através do nome escrito na escova e na tampa do estojo, o dentifrício utilizado foi a pasta; kit 2 - estojo coletivo feito em lona $(40 \mathrm{~cm} / 20$ $\mathrm{cm})$, para 0 armazenamento existem casulos para guardar as escovas ea pasta (uso col etivo), a identificação da escova é feita através de etiqueta; kit 3 - estojo coletivo de plástico com tampa ( $28 \mathrm{~cm} / 18$ $\mathrm{cm} / 7 \mathrm{~cm}$ ), as escovas são identificadas através de etiqueta e estão separadas individualmente por sacos plásticos, o dentifrício éa pasta; kit 4 - estojo coletivo feito de garrafa PET, cortada a $12 \mathrm{~cm}$ do fundo, ou lata porta-lápis de $10 \mathrm{~cm}$ de comprimento por $9 \mathrm{~cm}$ de diâmetro, não tem separação entre as escovas e são identificadas por etiquetas, a pasta é coletiva; kit 5- suporte coletivo para porta-escova composto de plástico $(25 \mathrm{~cm} /$ $9 \mathrm{~cm} / 3 \mathrm{~cm}$ ), armazena porta-escovas e dentifrício líquido coletivo, porta-escovas (de plástico, 19,5 $\mathrm{cm} / 3 \mathrm{~cm}$ ) possuem orifícios para a circulação do ar, a identificação dos materiais é feita através de uma caneta marcadora definitiva .

\section{Primeira etapa}

Vinte avaliadores (amostra de conveniência), responsáveis pela higiene bucal dos escolares de Bauru e São José dos Campos - SP (professores de educação infantil e ensino fundamental, e por cirurgiões dentistas), responderam a dois questionários (Anexos I e II) referentes às condições de armazenamento, higieneepraticidade doskitsque utilizaram. 
A análise estatística foi realizada por meio do teste Wilcoxon com $p<0,05$.

\section{Segunda etapa}

178 escolares de 4 a 8 anos de Bauru e Bariri, São Paulo participaram deste estudo (amostra deconveniência). Cadacriança aplicou uma quantidade de pasta (Sorriso, Colgate-Palmolive, São Paulo - SP, 1100 ppmF) e "dentifrício líquido" (Dentics, Poli Produtos, Lorena- SP, 1100 ppmF), para a prática da escovação. Nenhuma criança recebeu orientação no momento da aplicação. Vale ressaltar que, na cidade de Bauru, as crianças foram assistidas pelos acadêmicos da Faculdade de Odontologia deBauru - USP. A título de entendimento, o termo "dentifrício líquido" será para se diferenciar do creme dental (não é tão líquida como a água e nem tão sólido como as pastas tradicionais). A pesagem foi realizada em triplicata para ambos os dentifrícios através de uma balança portátil de precisão (Figura 1), sendo encontrada uma média, a qual foi utilizada neste estudo. Também através de método observacional analisou-se a forma como os escolares dispensavam os dentifrícios nas escovas.

A análise estatística foi obtida através da comparação das médias das quantidades dispensadas nas cidades e o tipo de dentifrício utilizado. 0 coeficientedecorrelação de Pearson foi utilizado para verificar a correlação entre a idade e a quantidade de dentifrício dispensada. Para comparar as médias da quantidade nas cidades e 0 tipo de dentifrício usado, foi utilizada a análise de covariância, sendo a idade como covariável, com $\mathrm{p}<0,05$.

\section{Resultados}

0 kit 5 obteve graus de satisfeito emuito satisfeito quando comparado aos kits 1 a 4 (pouco satisfeito e insatisfeito) (Figura 2).

$\mathrm{Na}$ comparação direta entre os kits, o kit 5 demonstrou ter um custo bastante acessível, encontrando-se entre os mais baratos.

A quantidade de pasta de dente dispensada pelos escolares foi em média 0,41g em Bauru e $0,48 \mathrm{~g}$ em Bariri. Este resultado ocorreu principalmente devido às orientações dos acadêmicos para as crianças de Bauru. Não houve diferença estatística entre os escolares de Bauru eBariri em relação ao dentifrício líquido, sendo a média dispensada pela amostra de $0,15 \mathrm{~g}$.
Além disso, verificou-se correlação estatisticamente significante em relação à quantidade de pasta de dente dispen sada pelos escolares ea idade $(r=0,20 p=0,005)$. Em relação ao dentifrício líquido, não houvecorrelação estatisticamentesignificante. Isto demonstra grandevariabilidade na administração do dentifrício na forma de pasta, sendo que quanto maior a idade, maior a quantidade. Com o dentifrício líquido, ocorreu uma padronização, pois através da "técnica da gota" (Figura 3), uma pequena quantidade pode ser facilmente dispensada. Verificou-se também que $28,1 \%$ da amostra utilizaram uma quantidade de pasta de dentemaior do que $0,50 \mathrm{~g}$, isto é, risco de desenvolver a fluorose dentária. Com o dentifrício líquido, 100\% da amostra aplicou uma quantidade menor do que a dose limite (Figura 3).

\section{Discussão}

Os governantes e os profissionais ligados à saúde e educação devem ter em mente que a prática da saúde bucal coletiva éal go rel evante, que deve ser tratada com a devida atenção. Para o sucesso dos programas, a preocupação não se resume apenas no fornecimento do material, mas em toda a logística da atividade.

A escova dentária, instrumento indispensável para a manutenção da saúde, deve ser armazenada em local adequado, e não como ferra-

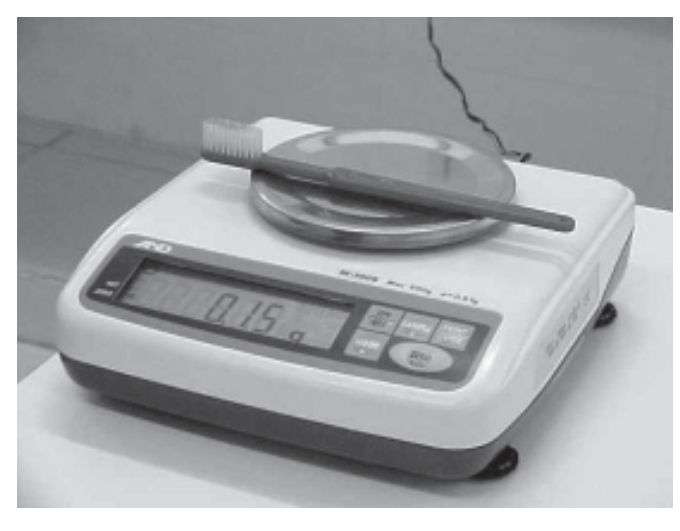

Figura 1. Balança portátil de precisão. 


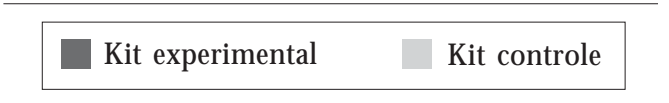

Armazenamento

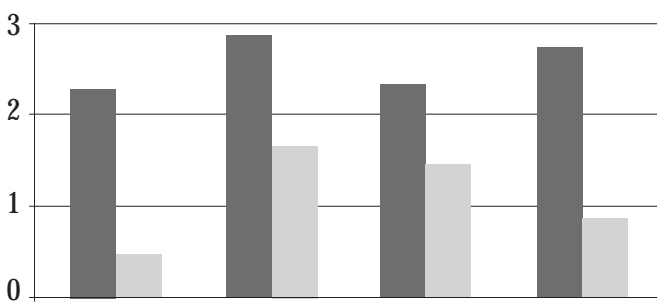

kit 5/kit 1 kit 5/kit 2 kit 5/kit 3 kit 5/kit 4

Higiene

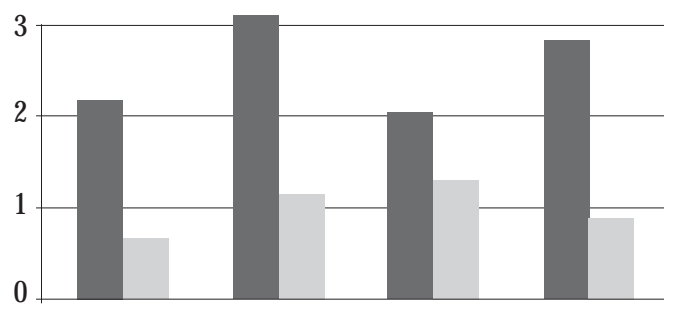

kit 5/kit $1 \quad$ kit 5/kit 2 kit 5/kit 3 kit 5/kit 4

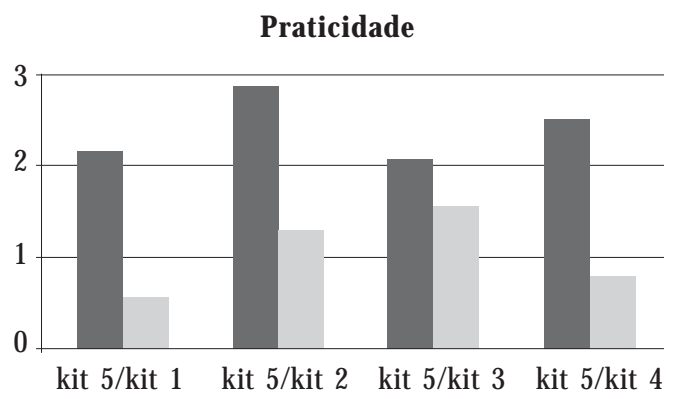

Figura 2. Resultado das comparações entre os Kits.

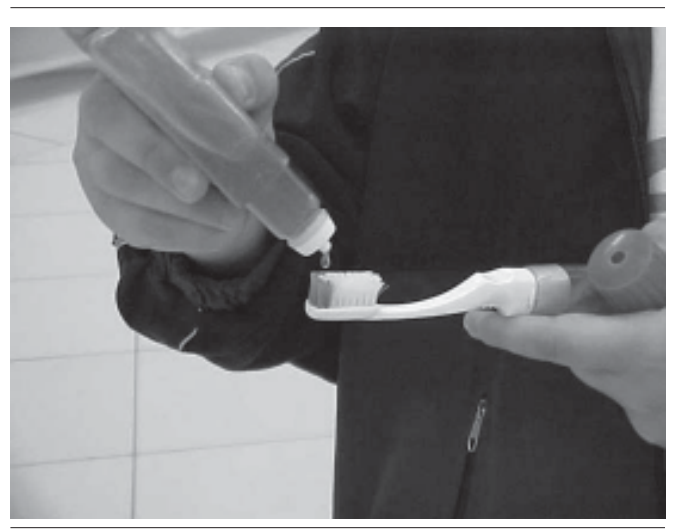

Figura 3. Técnica da gota. menta tipo chave de fenda (kit 2), ou armazenada em porta-lápis ou garrafa de refrigerante cortada ao meio, exposta a todo tipo de contaminação (kit 4). Muito menos dentro de saquinhos ou embalagens fechados sem circulação dear (kits 1 e 3), transformando as escovas em verdadeiros depósitos de microrganismos. Em adição, programas baseados unicamente na distribuição de escovas comprometem a relação custo-benefício, visto que as mesmas são acondicionadas de forma inadequada, muitas vezes perdidas, comprometendo a higiene bucal diária na escola.

0 kit ideal para a prática de higiene bucal coletiva deve atender as normas de vigilância sanitária, ter um suporte coletivo para armazenamento de todo o material. As escovas devem ser secas e mantidas em recipientes individuais fechados e com ventilação, tal qual preconizado por Dayoub et al. ${ }^{16}$ para evitar a contaminação. 0 kit experimental 5 demonstrou atender os requisitos citados, contribuindo para a prática de ações resolutivas em saúde bucal coletiva.

O flúor, um insumo farmacêutico, ésem dúvida um grande colaborador dos profissionais desaúde bucal, pois sua possi bilidade preventiva contra as cáries e sua utilização em larga escala beneficiaram milhões de pessoas. Contudo, a continuidade do uso do $\mathrm{F}$ em ações de saúde pública necessita cada vez mais atenção, pois o risco de ocorrência de fluorose dentária pode chegar a níveisinaceitáveis ${ }^{5}$.

Dados mais recentes do último levantamento das condições de saúde bucal da população brasileira, o SB - Brasil 2003 ${ }^{17}$, mostraram que a prevalência da fluorose foi de $9 \%$ das crianças de 12 anos e de $5 \%$ dos adolescentes de 15 a 19 anos no Brasil. Para a idade de 12 anos, os maiores índices foram encontrados nas regiões Sudeste e Sul (em torno de $12 \%$ ), enquanto que os menores nas regiões Centro-O este e N ordeste (cerca de 4\%).

Um estudo realizado por Lima et al. ${ }^{15} \mathrm{com}$ crianças brasileiras de 20-30 meses de idade residentes em áreas com água fluoretada mostrou que as mesmas atingiram em média $0,09 \mathrm{mg}$ de $\mathrm{F} / \mathrm{Kg} /$ dia, tendo a dieta contribuído com $45 \%$ eo dentifrício com $55 \%$, deste modo excedendo a dose limite quefica entre 0,05 a $0,07 \mathrm{mg}$ de $/ \mathrm{Kg} /$ dia. 0 estudo de Almeida et al. ${ }^{18}$, também com crianças brasileiras entre um e três anos, encontrou uma ingestão diária total de $\mathrm{F}$ pela dieta e dentifrício de 0,13 $\mathrm{mg} / \mathrm{Kg}$ peso corporal, sendo queo dentifrício contribuiu com $80 \%$ desta dose.

Uma boa alternativa para reduzir e dosar a quantidade de dentifrício dispensada na escova é a técnica transversal de aplicação do dentifrício ${ }^{19}$. 
Esta técnica consiste em posicionar o tubo de dentifrício em posição perpendicular ao longo eixo da escova, passar no centro da ponta ativa do instrumento uma quantidade de dentifrício correspondente a, no máximo, metade da largura da ponta ativa. Essa quantidade equivale a um grão de ervilha pequena, que é suficiente para a finalidade.

Para as crianças menores de quatro anos, sugere-se a "técnica da tampa", a qual consiste em, com a bisnaga fechada, pressionar levemente 0 tubo demodo a que fique retida, na parte interna da tampa (seja ela rosqueável ou não) uma pequena quantidade de pasta. Então, abre-seo tubo epressiona seaponta ativa da escova contra parte interna da tampa de modo a transferir para a escova a pequena quantidade de pasta ali retida ${ }^{20}$.

0 dentifrício deve ser aplicado de forma que o bico do tubo não tenha contato com as cerdas das escovas, evitando a contaminação, e também deve ser dosado para evitar o risco de fluorose dentária. 0 dentifrício na forma líquida com bico dosador se enquadra perfeitamente a estas características, solução esta descrita por Vilhena et al. ${ }^{21}$, que preconizaram 0 uso de dentifrício líquido através da "técnica da gota" para as ações de higienebucal coletiva.

Sendo assim, os resultados permitem afirmar que o kit experimental 5 foi o único a oferecer condições adequadas de armazenamento, administração e uso do material de higiene bucal coletiva.

\section{Colaboradores}

FV Vilhena trabalhou na concepção, pesquisa, metodologia e na redação final; SH C Sales Peres trabal hou na concepção, metodologia ena redação final; M L Caldana trabal hou na concepção e metodologia e M AR Buzalaf trabalhou na concepção e redação final. 
Anexol

ESCOLA:

CIDADE:

SÉRIE:

PERÍODO:

TEL. CONTATO:

KIT:

AVALIADOR:

Avalie os itens dos quadros abaixo conforme o seguinte código:
MS - MUITO SATISFEITO
S - SATISFEITO
PS - POUCO SATISFEITO
I - INSATISFEITO
1) Armazenamento
MS S PS I

1.1 - Da escova dental dos alunos

1.2 - Do dentifrício dos alunos

1.3 - Do kit todo na escola

2) Condições dehigiene (acúmulo deágua eresíduos) MS S PS I

2.1 - Da escova dental dos alunos

2.2 - Do dentifrício dos alunos

2.3 - Do kit na escola

3) Praticidade

MS S PS I

3.1 - No design

3.2 - Na limpeza do kit

3.3 - No armazenamento na escola

3.4 - No armazenamento da escova dental do aluno

3.5 - No armazenamento do dentifrício do aluno

3.6 - Na utilização do kit no dia-a-dia

3.7 - Na colocação e dosagem do dentifrício para cada aluno

Anexoll

ESCOLA:

SÉRIE:

CIDADE:

TEL, CONTATO

PERÍODO:

KIT:

AVALIADOR:

Assinale o kit com o melhor resultado encontrado para cada item:

4) Armazenamento

Kit: $\quad$ Kit 5

Da escova dental dos alunos

Do dentifrício dos alunos

Do kit todo na escola 
5) Condições dehigiene (acúmulo de água e resíduos )

Kit: Kit 5

Da escova dental dos alunos

Do dentifrício dos alunos

Do kit na escola

6) Praticidade

Kit: $\quad$ Kit 5

No design

Na limpeza do kit

No armazenamento na escola

No armazenamento da escova dental do aluno

No armazenamento do dentifrício do aluno

$\mathrm{Na}$ utilização do kit no dia-a-dia

$\mathrm{Na}$ colocação e dosagem do dentifrício para cada aluno

\section{Referências}

1. Frazão $P, N$ arvai PC. Promoção de saúde bucal em escolas. In: Universidade de São Paulo, Faculdade de Saúde Pública, Disciplina de odontologia preventiva e saúde pública. M anual do aluno. São PauIo: Departamento de Prática de Saúde Pública; 1996.

2. Moysés ST, Watt R. Promoção de saúde bucal. Definições. In: Buischi YP, organizador. Promoção de saúde bucal na clínica odontológica. São Paulo: Artes M édicas; 2000.

3. Pinto VG. Prevenção da cárie dentária: dentifrícios com flúor. In: Pinto VG, organizador. Saúde bucal coletiva. $4^{\text {a }}$ ed. São Paulo: Editora Santos; 2000. p. 353-395.

4. Vilhena FV. Odontologia escolar. Bauru: S.E.; 1998.

5. Narvai PC. Cárie dentária e flúor: uma relação do século XX. Cienc Saude Colet 2000; 5(2): 381-392.

6. Brandão LMS, Coelho RS, Silveira JLGC. Avaliação do uso e acondicionamento de escovas dentárias em creches. Pesq Bras O dontoped Clin Integr 2001; $1(2): 37-41$

7. Chaves SCL, Vieira-da-Silva LMA. Efetividade do dentifrício fluoretado no controle da cárie dental: uma meta-análise. Rev. Saúde Pública 2002; 36(5):598606.

8. Conrado CA, M anganoti J, Yokoyama M C, Bottazzaro Jr A. Efeito de um programa educacional direto relacionado com a saúde bucal de escolares do município de Maringá, PR. Odontol Clín 1997; 7:21-30.

9. Dusablon MJ, Vincent JR. Toothbrush holder for a day-care center. J Dent Que 1989; 26:55-59.

10. Buzalaf MAR, Cury JA, Whitford GM. Fluoride exposures and dental fluorosis: a literature review. Rev Fac Odontol Bauru 2001; 9(1/2):1-10.

11. Buzalaf MAR, Bastos JRM, Lauris JRP, Almeida BS, Aquilante AG. Association between the early use of toothpaste and other variables with dental fluorosis: a transversal retrospective study. Rev Fac 0 dontol Bauru 2002; 10(3):196-200.

12. Cury JA. Determination of appropriate exposure of fluoride in non-EME countries in the future. J dent Res 2000; 79(4):901-906.
13. M ascarenhas AK, Burt BA. Fluorosis risk from early exposure to fluoride toothpaste. Comm Dent Oral Epidemiol 1998; 26(4):241-248.

14. Pessan JP, Silva SM B, Buzalaf MAR. Evaluation of the total fluoride intake of 4-7-year-old children from diet and dentifrice. J Appl Oral Sci 2003; 11:150-156.

15. Lima YBO, Cury JA. Ingestão de flúor por crianças através da água e dentifrício. Rev. Saúde Pública 2001; 35(6):576-581.

16. Dayoub MB, Rushito D, Gross A. Microbial contamination of toothbrushes. J Dent Res 1977; 56 (6):706.

17. Brasil. Ministério da Saúde. Secretaria de Políticas Especiais de Saúde. Área Técnica de Saúde Bucal. Levantamento epidemiológico em saúde bucal. Disponível em: http://www.datasus.gov.br/cgi/sbu$\mathrm{cal} / \mathrm{sbdescr.htm}$

18. Almeida BS, Cardoso VES, Buzalaf MAR. Fluoride ingestion from toothpaste and diet in 1-3-year-old Brazilian children. Comm Dent 0 Epidemiol 2007; 35:53-63.

19. Villena RS. An investigation of the transverse technique of dentifrice application to reduce the amount of fluoride dentifrice for young children. Ped Dent 2000; 22(4):312-317.

20. São Paulo. Secretaria de Estado da Saúde. Recomendações sobre o uso de produtos fluorados no âmbito do SUS/SP em função do risco de cárie, São Paulo, 2000. Disponível em: http://www.saude.sp.gov.br 21. Vilhena FV, Sales Peres SH C, Bastos JRM. Proposta de um novo kit para armazenamento e distribuição de escovas de dente e dentifrício para escolares. Odontologia e Sociedade 2004; 6(1):53.

Artigo apresentado em 21/10/2006 Aprovado em 27/06/2007 